\title{
DRP1-Mediated Mitochondrial Fission Regulates the Lung Epithelial Response to Allergen
}

\section{Sierra Bruno}

University of Vermont College of Medicine https://orcid.org/0000-0003-0764-9585

\section{Amit Kumar}

University of Vermont College of Medicine

\section{Zoe Mark}

University of Vermont College of Medicine

\section{Ravishankar Chandrasekaran}

University of Vermont College of Medicine

\section{Emily Nakada}

University of Vermont College of Medicine

Nicolas Chamberlain

University of Vermont College of Medicine

\section{Bethany Mihavics}

University of Vermont College of Medicine Joseph Walzer

University of Vermont College of Medicine Jonathon Cahoon

University of Vermont College of Medicine

Anne E. Dixon

University of Vermont College of Medicine

\section{Brian Cunniff}

University of Vermont College of Medicine

Vikas Anathy ( $\nabla$ vikas.anathy@med.uvm.edu )

University of Vermont College of Medicine

\section{Research}

Keywords: Mitochondrial fission, Epithelial cell, HDM, allergic airway disease, DRP1

Posted Date: June 29th, 2021

DOI: https://doi.org/10.21203/rs.3.rs-654294/v1 
License: (c) (i) This work is licensed under a Creative Commons Attribution 4.0 International License. Read Full License 


\section{Abstract}

Background. Mitochondria regulate a myriad of cellular needs and functions. Dysregulation of mitochondrial control within airway epithelial cells has been implicated in the pro-inflammatory response to allergens in asthmatics. Because of their multifaceted nature, mitochondrial structure needs to be tightly regulated through fission and fusion. Dynamin Related Protein 1 (DRP1), a cytosolic GTPase, is a key driver of mitochondrial fission. During allergic asthma, airway epithelial mitochondria appear smaller and structurally altered. The role of DRP1-mediated mitochondrial fission, however, has not been fully elucidated in allergic airway disease.

Methods. We used a Human Bronchial Epithelial Cell line (HBECs), primary Mouse Tracheal Epithelial Cells (MTECs), and conditional ablation of DRP1 in lung epithelial cells to investigate mitochondrial fission and its impact on the pro-inflammatory response to House Dust Mite (HDM) in vitro and in vivo.

Results. Our data suggest that, following HDM challenge, mitochondrial fission is rapidly upregulated in airway epithelial cells and precedes production of pro-inflammatory cytokines and chemokines. Further, deletion of DRP1 in lung epithelial cells lead to decreased mitochondrial fission and enhanced proinflammatory signaling in response to HDM. Analysis of lung epithelial specific DRP1 deletion in mice demonstrated enhanced Airway Hyper Responsiveness (AHR), inflammation, differential mucin transcription, and epithelial cell death.

Conclusions. Mitochondrial fission is rapidly upregulated in airway epithelial cells following HDM exposure, prior to epithelial release of pro-inflammatory cytokines and chemokines. Deletion of DRP1, a necessary pro- fission protein, reduces fission and enhances the pro-inflammatory epithelial response to $\mathrm{HDM}$, exacerbating the allergic response.

\section{Background}

Asthma is an inflammatory respiratory disease that is estimated to affect over 339 million people globally (1). In roughly $50-60 \%$ of asthma patients, complex allergens such as House Dust Mite (HDM) exacerbate the symptoms (2). Epithelial cell mitochondria play a key role in the pathogenesis and progression of asthma and the allergic airway response (3-6). It has long been documented that lung epithelial cells of asthmatics and mouse models of asthma have altered mitochondria with damaged cristae $(7,8)$. Recent studies have demonstrated that allergen-induced alterations in mitochondrial function drives epithelial pro-inflammatory signaling, apoptosis, and enhance AHR $(3,8-10)$.

Structural changes to mitochondria that influence function, i.e. fission and fusion, are integral for mammalian cellular homeostasis and survival (11-17). One of the key proteins necessary for mitochondrial fission is the GTPase Dynamin Related Protein 1 (DRP1). DRP1 is a cytosolic protein that, upon activation via phosphorylation and/or other post-translational modifications at key residues, localizes to the mitochondrial outer membrane at sites where the endoplasmic reticulum interacts with and pre-constricts the mitochondrion. There, it interacts with different outer mitochondrial membrane- 
bound proteins, including FIS1 (mitochondrial FISsion protein 1), MFF (Mitochondrial Fission Factor), MiD49 (Mitochondrial Dynamics protein 49) and MiD51 (Mitochondrial Dynamics protein 51), to organize into multimeric rings around the mitochondria and constrict the outer mitochondrial membrane to allow the separation of mitochondria into two segments (fission) (18-22). Several studies have suggested that mitochondria appear visually smaller in the airway epithelia of asthmatics or mouse models of asthma, seen via transmission electron microscopy $(7,8)$, but the role of epithelial DRP1-mediated mitochondrial fission has yet to be fully elucidated in the allergic airway disease.

This study aimed to determine whether exposure to complex allergen induces mitochondrial fission and investigate the contribution of DRP1 mediated mitochondrial fission in the epithelial response to allergen. We characterized mitochondrial fission dynamics in airway epithelia after allergen exposure and elucidated potential mechanisms of action in regulation of the allergic response initiated by epithelial cells.

\section{Methods}

Study approval. All mouse studies were approved for use by the Institutional Animal Care and Use Committee of the University of Vermont under protocol number X9-016.

HDM. HDM (XPB70D3A2.5) was purchased from GREER and suspended in Phosphate Buffered Saline (PBS). HDM concentration was determined by protein concentration.

Human bronchial epithelial cell culture experiments. HBEC-3KTs (ATCC CRL-4051) were plated in 2mL DMEM/F12 (Gibco 11330057) with added growth factors at 4 X 106 cells per dish in 35mm dishes and allowed to adhere overnight. Cells were then exposed to either 50 $\mathrm{gg}$ HDM or PBS control, after a 2-hour starvation period in DMEM/F12 without added growth factors. Cells were subsequently exposed to the same HDM concentration again 48 hours later, and supernatants and cell lysates were collected at corresponding time points. Cells used for immunofluorescence were plated on chamber slides (Nunc LabTek II CC2 Chamber Slide System 154852) at $2.5 \times 10^{6}$ cells per chamber and put through the same exposure protocol.

Primary mouse tracheal epithelial cell culture experiments. Primary MTECs were isolated and cultured, as previously described (23), from mice containing LoxP sites flanking exons 3 and 5 of Drp1. Drp 1 loxp/loxp mice were obtained from Dr. Hiroma Sesaki at Johns Hopkins University (13). MTECs were plated in $35 \mathrm{~mm}$ dishes coated with $100 \mu \mathrm{g}$ monomeric rat tail collagen (Corning 354236 ) at $7 \times 10^{6}$ cells per dish and allowed to adhere overnight. Cells were infected with adenovirus expressing Cre recombinase (Vector BioLabs 1700) at an MOI of 8 per cell to delete Drp 1 (adenovirus expressing GFP - Vector BioLabs 1300 - was used as a control). Cells were incubated for 3 to 5 days before being exposed to HDM in the same manner as described for the HBECs and supernatants and cell lysates were collected at corresponding time points. Cells used for immunofluorescence were plated on chamber slides coated with collagen at 4 $X 10^{6}$ cells per chamber and subjected the same adenoviral and HDM protocols. 
Transgenic mice. Bi-transgenic mice, obtained from Dr. Whitsett at Cincinnati Children's Hospital (24), were crossed with $\operatorname{Drp} 7^{10 x p / l o x p}$ mice. The bi-transgenic mice contained two genetic inserts, rat club cell secretory protein promoter linked to reverse tetracycline transactivator (CCSP-rtTA) and a tetracycline operon linked with Cre recombinase (TetOP-Cre). The resulting mice (CCSP-rtTA+, TetOP-Cre+, Drp $1^{\text {loxp/loxp }}$ ) could deplete DRP1 from club cells ( $\triangle E$ Epi-Drp1) upon induction by doxycycline, introduced via mouse chow (6 g/kg; Purina Diet Tech, St Louis, Mo). Mice were given doxycycline-containing chow 10 days before HDM exposure and were maintained on doxycycline-containing chow for the duration of the experiment. Littermates missing one of the three genetic inserts (CCSP-rtTA+/TetOP-Cre + or CCSP$r T A+/ D r p 1^{\text {loxp/loxp }}$ ) also given doxycycline-containing chow were used as controls (Ctrl).

AHR assessment. Mice were anesthetized using sodium pentobarbital $(90 \mathrm{mg} / \mathrm{kg})$ via intraperitoneal injection and tracheotomized using 18-guage cannula. Mice were mechanically ventilated at a rate of 200 breaths/minute using a FlexiVent computer-controlled small-animal ventilator (SCIREQ). Newtonian resistance $(\mathrm{Rn})$, tissue dampening $(\mathrm{G})$, and tissue elastance $(\mathrm{H})$ were measured in the mice after exposure to increasing concentrations of aerosolized methacholine. Results are shown as the average of three peak measurements with a COD value greater than 0.85 .

Bronchoalveolar lavage fluid processing. Bronchoalveolar lavage fluid (BALF) was collected by washing the airways with $1 \mathrm{~mL}$ of cold, sterile PBS. Cells were then isolated via centrifugation and total cell counts were determined by using a hemocytometer (Hausser Scientific). Cytospins were conducted and cells were stained using Hema3 stain reagents (Thermo Fisher Scientific) to obtain differential cell counts. 300 cells minimum were counted to determine differential counts.

ELISAs. For cell culture experiments, supernatants were collected 24 hours after the second HDM dose and used to assess secreted levels of IL8, IL6, CCL20, and G-CSF (R\&D Systems) per the manufacturer's instructions. For mouse experiments, right side lung lobes were flash frozen immediately after harvest and crushed to make lysates in buffer containing $137 \mathrm{mM}$ Tris-HCL (pH 8.0) $130 \mathrm{mM} \mathrm{NaCl}$, and 1\% NP40. Samples were normalized to total lung protein and used to assess expression levels of IL5, IL6, IL33, CXCL1, Eotaxin-1 (R\&D Systems DuoSet ELISA Kits), IL4, and IL13 (eBioscience) per manufacturer's instructions.

Western blots. For cell culture experiments, cells were lysed in buffer containing $137 \mathrm{mM}$ Tris-HCL (pH 8.0) $130 \mathrm{mM} \mathrm{NaCl}$, and 1\% NP-40. For mouse experiments lungs were flash frozen, pulverized and crushed and lysates were made in the same buffer. The desired soluble proteins were separated from insoluble proteins via ultracentrifugation. Following protein quantification, samples were resuspended in loading buffer with dithiothreitol (DTT) and resolved by SDS-PAGE. Proteins were transferred to PVDF and membranes were probed using standard immunoblotting protocol. Membranes were probed for phosphorylated DRP1 (pDRP1 - Cell Signaling Technology 3455S), total DRP1 (tDRP1 - BD 611113), and $\beta$-actin (Sigma A5441). Quantification of protein expression was determined by densitometry using ImageJ software (NIH, https://imagej.nih.gov/ij/). 
Immunofluorescence. For cell culture experiments, cells were fixed at corresponding time points following HDM exposure using freshly prepared $4 \%$ paraformaldehyde for 10 minutes and permeabilized for 30 minutes in $0.2 \%$ Tween-20 in PBS at room temperature. For mouse experiments, left lung lobes were formalin fixed overnight at $4^{\circ} \mathrm{C}$, mounted in paraffin, and sectioned at $5 \mu \mathrm{m}$ thickness. Sections were fixed to glass slides and prepared for immunofluorescence by deparaffinization using xylene and rehydration through a series of ethanol washes. Lung antigen retrieval was achieved by submersing slides in sodium citrate buffer $(\mathrm{pH} 6.0)$ with $0.05 \%$ TWEEN-20 at $95^{\circ} \mathrm{C}$ for 20 minutes. For both cells and lung sections, samples were blocked in $1 \%$ BSA in PBS for 1 hours, followed by overnight incubation on primary antibody, diluted in PBS, at $4^{\circ} \mathrm{C}$. For cells, pDRP1 was used at a concentration of 1:200 and VDAC (Invitrogen PA1-954A) was used at 1:300. For lung sections, CC10 (Santa Cruz sc-390313) and cleaved caspase-3 (Cell Signaling Technology 9661) were used at a concentration of 1:300. Slides were then washed 3 X 5 minutes in PBS and subsequently incubated for 1 hour at room temperature in fluorescentconjugated secondary antibodies (Life Technologies) at half the concentration of the primary antibodies in 1\% BSA in PBS. Samples were counterstained with DAPI at 1:4000 in 1\% BSA in PBS for 10 minutes at room temperature and mounted using AquaPoly mounting medium (Polysciences 18606). Images were taken on a Nikon Laser Scanning Confocal Microscope (Nikon A1R-ER). Brightness and contrast were adjusted equally for corresponding images and images were analyzed for mean fluorescence intensity (MFI) and for mitochondrial form factor ( $F F=$ perimeter $^{2} / 4 \pi^{*}$ area) using Image J software.

Analysis of mRNA expression. MUC5AC and MUC5B were quantified in mouse by extracting RNA from flash frozen, pulverized left lung lobes using TRIzol (Invitrogen 15596018). RNA was purified using the RNeasy kit (Qiagen). $1 \mu \mathrm{g}$ of RNA was reverse transcribed to cDNA (Promega) and SYBR Green Supermix (Bio-Rad) was used to quantify mRNA expression using RT-qPCR. For Drp1 quantification, lung epithelial cells were isolated from lungs of $\triangle E p i$-Drp1 mice on doxycycline diet for 10 days using the GentleMACS lung dissociation kit (Miltenyi Biotech) followed by the EasySep mouse epithelial cell enrichment kit II (STEMCELL Technologies). Isolated epithelia were lysed in TRIzol and RNA was isolated and reverse transcribed in the same manner as whole lung lysates. Drp1 expression in MTECs was also quantified by RT-qPCR following TRIzol lysis. Expression values were normalized to the geometric mean of GAPDH, $P P 1$, and $R P 2$ using the $\triangle \triangle C T$ method. Sequences of primers used in this study are listed in Table 1.

Mucus metaplasia quantification. Paraffin-embedded $5 \mu \mathrm{m}$ tissue sections were mounted on slides, deparaffinized and rehydrated, and antigen retrieval was performed. PAS staining was conducted, and images were captured on a Leica VERSA8 whole slide imager. Mucus metaplasia was measured in the airways by measuring positive PAS-stained area using the Positive Pixel Count algorithm of Aperio ImageScope Software (Aperio Technologies).

Caspase assay. $25 \mu \mathrm{g}$ of tissue lysates were diluted to $25 \mu \mathrm{L}$ in $\mathrm{dH} 2 \mathrm{O}$ and incubated with $25 \mu \mathrm{L}$ CaspaseGlo 3/7 assay reagent (Promega) in an opaque plate in the dark at room temperature for 30 minutes. Total luminescence was measured using a Synergy HTX plate reader (Biotek) and values were recorded as relative activity. 
Microarray analysis. GEO2R (http://www.ncbi.nlm.nih.gov/geo/info/geo2r.html) was used to compare differentially expressed genes between moderate and severe asthmatics, as classified by the American Thoracic Society, and non-asthmatic controls on GSE43696 $(25,26)$. GEO2R performs a base 2-log transformation.

Statistics. Outliers were determined using the ROUT method in GraphPad Prism 8 with a $Q=2 \%$. The Shapiro-Wilk normality test was run. Normal data were analyzed by either two-tailed student's t-test or two-way ANOVA, accordingly. For two-way ANOVA analysis, Tukey's post-hoc test was used to adjust for multiple comparisons. If one or more of the groups did not pass the Shapiro-Wilk normality test, those data were analyzed by two-way ANOVA followed by 2-stage linear step-up procedure of Benjamini, Krieger, and Yekutieli to control for false discovery rate. A $p$-value $<0.05$ was considered significant. Data were averaged and expressed as the mean \pm SEM.

\section{Results}

Human and mouse airway epithelial cells upregulate DRP1-mediated mitochondrial fission in response to allergen. Previous literature suggests that in people with asthma, epithelial mitochondria have altered structure including smaller overall size $(7,8)$. We, thus, aimed to determine whether DRP1-mediated mitochondrial fission is induced upon airway epithelial stimulation with HDM. We retrospectively analyzed a microarray dataset (GSE43696) retrieved through the NCBI Gene Expression Omnibus (GEO) to determine basal transcription of Drp1 in bronchial epithelial cells of human patients with moderate and severe asthma, as described by the American Thoracic Society $(25,26)$. Expression of two transcript variants of DRP1, both expressed in most human cell types, is significantly upregulated in severe asthmatic bronchial epithelial cells compared to non-asthmatic controls (Fig. 1A). To further analyze the role of this upregulation, we assessed the activity of DRP1 in vitro in human bronchial epithelial cells (HBEs) exposed to HDM. HDM induces the secretion of pro-inflammatory cytokines and chemokines upregulated and released by epithelial cells 24 hours after allergen exposure (Fig. 1B). Phosphorylation of DRP1 at serine 616 (S616) has been shown to enhance GTPase activity of the protein $(11,27,28)$. We found that HDM exposure of HBEs acutely increases the phosphorylation of DRP1 at S616 at 40 minutes post HDM exposure and this phosphorylation dissipates within 120 minutes from HDM exposure (Fig. 1C, D). Mitochondrial fission initiated by activated DRP1 can be determined by measuring average mitochondrial size $(29,30)$. This can also be quantified by form factor, a measurement of length and branching of the mitochondrial network (31-33). Activation of DRP1 immediately precedes an induction of mitochondrial fission in HBEs exposed to HDM, as indicated by decreased form factor and average mitochondrial area at 120 minutes post-exposure (Fig. 1E, F). Next, to understand the role of mitochondrial fission in the pro-inflammatory response initiated by airway epithelial cells, we aimed to confirm these processes also occurred in primary mouse tracheal epithelial cells (MTECs). Upon stimulation with HDM, MTECs respond similarly to human airway epithelial cells. Phosphorylation of DRP1 is increased 40 minutes after HDM exposure that dissipates by 120 minutes (Figure S1A, B). As in human airway epithelial cells, activation of DRP1 precedes the pro-inflammatory response initiated by allergen stimulation (Figure S1C). 
In vitro deletion of DRP1 enhances the pro-inflammatory response to allergen. Considering DRP1mediated mitochondrial fission occurs prior to the pro-inflammatory response initiated in airway epithelial cells, we sought to determine the role of fission in pro-inflammatory signaling. We utilized a gene deletion system whereby insertion of two Loxp sites allows for cre recombinase-induced excision of a segment of DNA within the gene of interest. We determined that infecting MTECs containing Drp $7^{\text {loxp/loxp }}$ sites with an adenovirus vector expressing cre recombinase (Ad-Cre) at an $\mathrm{MOI}$ of 8 significantly decreased Drp 1 mRNA levels to $\sim 10 \%$ of adenoviral control (Ad-Null) levels (Fig. 2A). While HDM induced mitochondrial fission in control cells, stimulation with HDM of Drp 1-deleted MTECs does not induce a decrease in mitochondrial networking as determined by form factor, thus exhibiting an impairment in mitochondrial fission following Drp1 deletion (Fig. 2B, C). Using this system, we also analyzed the pro-inflammatory response of MTECs after deletion of Drp1. Drp1-deleted MTECs exposed to HDM exhibited a significant increase in the secretion of pro-inflammatory cytokines and chemokines 24 hours post exposure to HDM (Fig. 2D).

Conditional deletion of Drp1 in vivo increases inflammation in response to allergen exposure in a mouse model of allergic airways disease. To understand the impact of the enhancement of the epithelial proinflammatory response to HDM due to the deletion Drp1, we generated a CCSP-rTetA/TetOPCre/Drp $1^{\text {loxp/loxp }}$ mouse to delete Drp1 in lung epithelial (club) cells in the presence of doxycycline. Mice were put on diet containing doxycycline 10 days before initiation of the allergic airways disease experimental protocol and the diet was continued until the end of the sensitization and challenge protocol. Mice were sensitized on days 0 and 7 followed by a single challenge on day 14 with $25 \mu \mathrm{g} \mathrm{HDM}$, or PBS control, intranasally. Mice were sacrificed 4 hours after the single challenge to assess the role of DRP1 in initiation of the allergic airway response (Fig. 3A). Isolated lung epithelial cells revealed significant reduction of Drp1 transcript in the $\triangle E p i$-Drp 1 mice compared to littermate control (Ctr) mice. This reduction could not be seen, however, in whole lung lysate, confirming lung epithelial specific Drp1 deletion (Fig. 3B). The bronchoalveolar lavage fluid (BALF) was analyzed for immune cell infiltration into the lungs. This analysis revealed a significant increase in the total cell infiltrates to the lungs after HDM challenge with Drp1 deletion compared to littermate controls (Fig. 3C). Assessment of the cell types in the airways revealed eosinophils and lymphocytes were significantly upregulated in the lungs of Drp1-deleted mice challenged with HDM (Fig. 3D). Whole lung tissue lysates were analyzed for pro-inflammatory cytokines, chemokines and Th2 cytokines via ELISA. There were no differences seen in production of several epithelial-secreted pro-inflammatory cytokines such as IL-6 and IL-33 or Th2 cytokine IL5 between $\triangle E p i-D r p 1$ mice challenged with HDM, compared to HDM-challenged Ctrls. Alternatively, $\triangle E p i-D r p 1$ mice challenged with HDM expressed higher levels of the pro-inflammatory eosinophil chemokine Eotaxin, and the Th2 cytokines IL4 and IL13 (Fig. 3E). This could suggest a role for mitochondrial fission in regulation of specific epithelial pro-inflammatory signaling.

In vivo conditional deletion of Drp1 enhances mucin expression and epithelial apoptosis.

A common hallmark of allergen-induced asthma and an indicator of asthma severity is mucus metaplasia (34-36). We, therefore, examined mucin expression in the lungs after Drp1 deletion. While 
MUC5AC mRNA levels were not expressed at a higher level in $\triangle$ Epi-Drp1 mice compared to Ctrl HDM exposed mice, $M U C 5 B$, more commonly upregulated in fibrotic lung diseases and linked with severity of fibrosis (37-40), was significantly increased in both PBS and HDM groups compared to corresponding Ctrl groups (Fig. 4A). We also examined mucin secretion into the BALF via ELISA. MUC5AC secretion was significantly enhanced in the HDM-exposed $\triangle E p i$-Drp1 mice compared the HDM-exposed $C t r /$ mice. MUC5B secretion, though not significant, trended higher in the $\triangle E p i-D r p 1$ mice compared to Ctrls (Fig. 4B). And mucus staining trended higher but was not significantly increased in the airways of $\triangle E p i-$ Drp1 mice compared to littermate controls as demonstrated by Periodic Acid Schiff (PAS) (Fig. 4C, D). This data suggests epithelial DRP1 may regulate differential mucin expression, further suppressing severity of the allergic response to HDM.

Mitochondrial fission is believed to play a key role in cell death regulation (41-43), therefore we examined activation of the apoptosis regulator, Caspase-3 in mouse lung tissue. Immunofluorescence for active (cleaved) caspase-3 was conducted and analyzed, revealing an increased level of cleaved caspase3 in the airway epithelia of $\triangle E p i-D r p 1$ mice compared to littermate controls challenged with HDM, as indicated by increased relative mean fluorescence intensity (Fig. 5E, F). Further, we performed a CaspaseGlo assay in mouse lung tissue and observed a significant increase in the activity of Caspases in tissue lysates of $\triangle E$ Epi-Drp1 mice compared to littermate controls (Fig. 5G), a readout of increased apoptosis. This data together indicates a worsened allergic airway response to HDM after Drp1 deletion with enhanced epithelial cell death and altered mucin profile.

Drp1 deletion in vivo enhances airway hyperresponsiveness to methacholine. To assess methacholineinduced airway hyperreactivity (AHR), additional experiments were carried out with two challenges to allow more time to develop reactivity in the lungs (Fig. 5A). While Ctrl mice sensitized and challenged with HDM did not have increased AHR (i.e. Rn, G, and H) as compared to PBS sensitized and challenged mice, $\triangle E$ Ei-Drp 1 mice had significantly increased AHR in response to HDM (Fig. 5B). This data pattern could indicate increased inflammation and/or mucosal secretions inducing a collapse in the peripheral airways, as seen in other models of acute allergen -induced inflammation (44). This data suggests a more severe response to allergen following Drp1 deletion.

\section{Discussion}

We found that both human and mouse airway epithelial cells rapidly upregulate the phosphorylation of DRP1 at Serine 616 (S616), indicating a role in early response to the allergen. Drp1 deletion in vitro and in vivo hindered mitochondrial fission and enhanced secretion of pro-inflammatory markers from epithelial cells following HDM exposure as well as the activation of pro-apoptotic markers. Drp1 epithelial deletion also increased AHR in mice, a readout of airway remodeling during response to allergen insult. Finally, Muc5B transcription and apoptosis were also increased following Drp1 epithelial deletion in HDMchallenged mice. Together, these data demonstrate a role for DRP1-dependent mitochondrial fission in the regulation and response of airway inflammation initiated by airway epithelia in response to complex allergen. Increases in DRP1 expression and activity, and mitochondrial structural alterations, have already 
been linked to airway inflammatory responses to various insults in multiple cell types (45-49). The role DRP1 plays in initiation of the epithelial response to the complex allergen HDM, however, had not been previously demonstrated. Therefore, we have presented data evaluating the role of DRP1, and mitochondrial fission, in the initiation of the airway allergic response to the complex allergen HDM in both in vitro and in vivo model systems.

Several studies have linked DRP1-mediated mitochondrial fission to activation of NF-KB, thus linking increased DRP1 expression with an enhanced inflammatory response to various stimuli in multiple cell types (50-52). There is, however, a lack in understanding of the role DRP1 plays in inflammation initiated by airway epithelial cells in response to allergen. Though several studies have suggested DRP1 induces inflammatory signaling, here we show a link between DRP1 and suppression of HDM-induced proinflammatory cytokine and chemokine release by airway epithelia in culture. In agreement with our work, another study demonstrated DRP1 suppresses pro-inflammatory signaling in macrophages stimulated with LPS via regulation of release of mitochondrial damage-associated molecular patterns (DAMPs) (53). Mitochondrial DAMPs play an important role in inflammatory signaling in response to lung injury (5456). Mitochondrial DAMP release could be suppressed by DRP1-induced fission in airway epithelia following HDM exposure, though more detailed studies are needed to examine this phenomenon. This scenario represents a signal-dependent dichotomous action of DRP1 in regulation of inflammatory signaling.

Mucus metaplasia can contribute to enhanced AHR (34-36). Mucus is secreted by goblet cells, a specific subset of epithelial in the airways (57). Our data suggest the club cell specific deletion of Drp 1 increases mucin transcription and translation after HDM exposure. Club cells are known to act as progenitor-type cells and can differentiate into numerous epithelial subtypes, including goblet cells (58). Mitochondrial fission is also known to play a role in regulation of differentiation of various cell types (59-61). This data suggest a link between mitochondrial fission and differentiation of club cells into pathological epithelial subtypes.

DRP1-mediated mitochondrial fission plays regulates programmed cell death as well as cell survival in stress environments $(31,43,62-67)$. DRP1 expression and mitochondrial fission have been linked to mitophagy of mitochondria damaged by oxidative stress and decrease in apoptosis $(68,69)$. A recent publication also suggests that cockroach allergen induces mitophagy in airway epithelia (70).

Additionally, the literature demonstrates that HDM induces reactive oxygen species (ROS) production and oxidative stress and damage of airway epithelia (71-74). Our data indicate DRP1 balances epithelial cell survival in response to HDM exposure, as indicated by increased cleavage and activity of caspase 3 after Drp1 deletion. This could suggest attenuated clearance of damaged mitochondria following Drp1 deletion. DRP1-mediated mitochondrial fission facilitates clearance of damaged mitochondrial segments and reduces various stresses, thus suppressing cell death and protecting against barrier damage, though more mechanistic studies in the future could reveal the delicate balance of DRP1 action in allergic airway disease. 


\section{Conclusions}

Collectively, our data indicate that DRP1-mediated mitochondrial fission is important for regulation of the pro-inflammatory response by airway epithelia as well as airway epithelial survival after exposure to HDM. This information and more mechanistic studies in the future will reveal the complex role of DRP1 in allergic airway disease.

\section{Abbreviations}

DRP1: Dynamin Related Protein 1; HBECs: Human Bronchial Epithelial Cells; MTECs: Mouse Tracheal Epithelial Cells; HDM: House Dust Mite; AHR: Airway Hyper Responsiveness; PBS: Phosphate Buffered Saline; $\triangle E$ Epi-Drp1: Epithelial-specific deletion of Drp1; BALF: BronchoAlveolar Lavage Fluid; ELISA; Enzyme-Linked ImmunoSorbent Assay

\section{Declarations}

Ethics approval and consent to participate: All mouse studies were approved for use by the Institutional Animal Care and Use Committee of the University of Vermont under protocol number X9-016.

Consent for publication: Not applicable

Availability of data and materials: The human microarray dataset analyzed during the current study are available in the Gene Expression Omnibus (GEO) through

$\mathrm{NCBI}$, https://www.ncbi.nlm.nih.gov/geo/query/acc.cgi?acc=GSE43696. ${ }^{[25,26]}$ All other data generated or analyzed are included in this publication or the supplementary information files.

Competing interests: The authors declare that they have no competing interests.

Funding: This work is supported by NIH R01s HL122383, HL136917, and HL141364 to VA, and by a NIHT32 training grant HL076122 fellowship to SRB.

Authors Contributions: SRB conducted all experiments for and wrote this manuscript. AK assisted in western blot analysis and conceptual discussion. ZM assisted in breeding of mice and conducting all mouse experiments. RC, EN, and NC assisted in conceptual discussion. BM aided in breeding of mice. JW helped with western blot analysis. JC helped establish culture techniques for and initiate this study. AED assisted in conceptual discussion. BC and VA assisted in conceptual discussion and experimental design and were major contributors to the editing of this manuscript. All authors read and approved the final manuscript.

Acknowledgements: The authors would like to acknowledge the Microscopy Imaging Center and the Vermont Lung Center at the University of Vermont Larner College of Medicine for their assistance in training and project discussions. 


\section{References}

1. Global regional. and national incidence, prevalence, and years lived with disability for 328 diseases and injuries for 195 countries, 1990-2016: a systematic analysis for the Global Burden of Disease Study 2016. Lancet. 2017;390(10100):1211-59.

2. Pakkasela J, Ilmarinen P, Honkamäki J, Tuomisto LE, Andersén H, Piirilä P, et al. Age-specific incidence of allergic and non-allergic asthma. BMC Pulm Med. 2020;20(1):9.

3. Aguilera-Aguirre L, Bacsi A, Saavedra-Molina A, Kurosky A, Sur S, Boldogh I. Mitochondrial dysfunction increases allergic airway inflammation. J Immunol. 2009;183(8):5379-87.

4. Mabalirajan U, Rehman R, Ahmad T, Kumar S, Leishangthem GD, Singh S, et al. 12/15-lipoxygenase expressed in non-epithelial cells causes airway epithelial injury in asthma. Sci Rep. 2013;3:1540.

5. Simoes DC, Psarra AM, Mauad T, Pantou I, Roussos C, Sekeris CE, et al. Glucocorticoid and estrogen receptors are reduced in mitochondria of lung epithelial cells in asthma. PLoS One. 2012;7(6):e39183.

6. Zifa E, Daniil Z, Skoumi E, Stavrou M, Papadimitriou K, Terzenidou M, et al. Mitochondrial genetic background plays a role in increasing risk to asthma. Mol Biol Rep. 2012;39(4):4697-708.

7. Konrádová V, Čopová C, Suková B, Houštěk J. Ultrastructure of the bronchial epithelium in three children with asthma. Pediatr Pulmonol. 1985;1(4):182-7.

8. Mabalirajan U, Dinda AK, Kumar S, Roshan R, Gupta P, Sharma SK, et al. Mitochondrial structural changes and dysfunction are associated with experimental allergic asthma. J Immunol. 2008;181(5):3540-8.

9. Kim SR, Kim DI, Kim SH, Lee H, Lee KS, Cho SH, et al. NLRP3 inflammasome activation by mitochondrial ROS in bronchial epithelial cells is required for allergic inflammation. Cell Death Dis. 2014;5(10):e1498.

10. Xu W, Ghosh S, Comhair SA, Asosingh K, Janocha AJ, Mavrakis DA, et al. Increased mitochondrial arginine metabolism supports bioenergetics in asthma. J Clin Invest. 2016;126(7):2465-81.

11. Taguchi N, Ishihara N, Jofuku A, Oka T, Mihara K. Mitotic phosphorylation of dynamin-related GTPase Drp1 participates in mitochondrial fission. J Biol Chem. 2007;282(15):11521-9.

12. Ishihara N, Nomura M, Jofuku A, Kato H, Suzuki SO, Masuda K, et al. Mitochondrial fission factor Drp1 is essential for embryonic development and synapse formation in mice. Nat Cell Biol. 2009;11(8):958-66.

13. Wakabayashi J, Zhang Z, Wakabayashi N, Tamura Y, Fukaya M, Kensler TW, et al. The dynaminrelated GTPase Drp1 is required for embryonic and brain development in mice. J Cell Biol. 2009;186(6):805-16.

14. Chen $\mathrm{H}$, Chomyn A, Chan DC. Disruption of fusion results in mitochondrial heterogeneity and dysfunction. J Biol Chem. 2005;280(28):26185-92.

15. Chen H, McCaffery JM, Chan DC. Mitochondrial fusion protects against neurodegeneration in the cerebellum. Cell. 2007;130(3):548-62. 
16. Li Z, Okamoto K, Hayashi Y, Sheng M. The importance of dendritic mitochondria in the morphogenesis and plasticity of spines and synapses. Cell. 2004;119(6):873-87.

17. Chen H, Detmer SA, Ewald AJ, Griffin EE, Fraser SE, Chan DC. Mitofusins Mfn1 and Mfn2 coordinately regulate mitochondrial fusion and are essential for embryonic development. J Cell Biol. 2003;160(2):189-200.

18. Bleazard W, McCaffery JM, King EJ, Bale S, Mozdy A, Tieu Q, et al. The dynamin-related GTPase Dnm1 regulates mitochondrial fission in yeast. Nat Cell Biol. 1999;1(5):298-304.

19. Labrousse AM, Zappaterra MD, Rube DA, van der Bliek AM. C. elegans dynamin-related protein DRP-1 controls severing of the mitochondrial outer membrane. Mol Cell. 1999;4(5):815-26.

20. Smirnova E, Griparic L, Shurland DL, van der Bliek AM. Dynamin-related protein Drp1 is required for mitochondrial division in mammalian cells. Mol Biol Cell. 2001;12(8):2245-56.

21. Chang $C R$, Blackstone $C$. Dynamic regulation of mitochondrial fission through modification of the dynamin-related protein Drp1. Ann N Y Acad Sci. 2010;1201:34-9.

22. Lee H, Yoon Y. Mitochondrial fission and fusion. Biochem Soc Trans. 2016;44(6):1725-35.

23. Wu R, Smith D. Continuous multiplication of rabbit tracheal epithelial cells in a defined, hormonesupplemented medium. In Vitro. 1982;18(9):800-12.

24. Perl AK, Tichelaar JW, Whitsett JA. Conditional gene expression in the respiratory epithelium of the mouse. Transgenic Res. 2002;11(1):21-9.

25. Li X, Hawkins GA, Moore WC, Hastie AT, Ampleford EJ, Milosevic J, et al. Expression of asthma susceptibility genes in bronchial epithelial cells and bronchial alveolar lavage in the Severe Asthma Research Program (SARP) cohort. The Journal of asthma: official journal of the Association for the Care of Asthma. 2016;53(8):775-82.

26. Voraphani N, Gladwin MT, Contreras AU, Kaminski N, Tedrow JR, Milosevic J, et al. An airway epithelial iNOS-DUOX2-thyroid peroxidase metabolome drives Th1/Th2 nitrative stress in human severe asthma. Mucosal Immunol. 2014;7(5):1175-85.

27. Zheng X, Chen M, Meng X, Chu X, Cai C, Zou F. Phosphorylation of dynamin-related protein 1 at Ser616 regulates mitochondrial fission and is involved in mitochondrial calcium uniporter-mediated neutrophil polarization and chemotaxis. Mol Immunol. 2017;87:23-32.

28. Otera $\mathrm{H}$, Ishihara N, Mihara K. New insights into the function and regulation of mitochondrial fission. Biochim Biophys Acta. 2013;1833(5):1256-68.

29. Anding AL, Wang C, Chang TK, Sliter DA, Powers CM, Hofmann K, et al. Vps13D Encodes a UbiquitinBinding Protein that Is Required for the Regulation of Mitochondrial Size and Clearance. Curr Biol. 2018;28(2):287. :- 95.e6..

30. Mizuno M, Kuno A, Yano T, Miki T, Oshima H, Sato T, et al. Empagliflozin normalizes the size and number of mitochondria and prevents reduction in mitochondrial size after myocardial infarction in diabetic hearts. Physiol Rep. 2018;6(12):e13741. 
31. Catanzaro MP, Weiner A, Kaminaris A, Li C, Cai F, Zhao F, et al. Doxorubicin-induced cardiomyocyte death is mediated by unchecked mitochondrial fission and mitophagy. Faseb j. 2019;33(10):11096108.

32. Antony PMA, Kondratyeva O, Mommaerts K, Ostaszewski M, Sokolowska K, Baumuratov AS, et al. Fibroblast mitochondria in idiopathic Parkinson's disease display morphological changes and enhanced resistance to depolarization. Sci Rep. 2020;10(1):1569.

33. Koopman WJ, Visch HJ, Verkaart S, van den Heuvel LW, Smeitink JA, Willems PH. Mitochondrial network complexity and pathological decrease in complex I activity are tightly correlated in isolated human complex I deficiency. Am J Physiol Cell Physiol. 2005;289(4):C881-90.

34. Dunican EM, Elicker BM, Gierada DS, Nagle SK, Schiebler ML, Newell JD, et al. Mucus plugs in patients with asthma linked to eosinophilia and airflow obstruction. J Clin Invest. 2018;128(3):9971009.

35. Evans CM, Williams OW, Tuvim MJ, Nigam R, Mixides GP, Blackburn MR, et al. Mucin is produced by clara cells in the proximal airways of antigen-challenged mice. Am J Respir Cell Mol Biol. 2004;31(4):382-94.

36. de Marco R, Marcon A, Jarvis D, Accordini S, Almar E, Bugiani M, et al. Prognostic factors of asthma severity: a 9-year international prospective cohort study. J Allergy Clin Immunol. 2006;117(6):124956.

37. Woodruff PG, Modrek B, Choy DF, Jia G, Abbas AR, Ellwanger A, et al. T-helper type 2-driven inflammation defines major subphenotypes of asthma. Am J Respir Crit Care Med. 2009;180(5):388-95.

38. Lachowicz-Scroggins ME, Finkbeiner WE, Gordon ED, Yuan S, Zlock L, Bhakta NR, et al. Corticosteroid and long-acting ß-agonist therapy reduces epithelial goblet cell metaplasia. Clin Exp Allergy. 2017;47(12):1534-45.

39. Hancock LA, Hennessy CE, Solomon GM, Dobrinskikh E, Estrella A, Hara N, et al. Muc5b overexpression causes mucociliary dysfunction and enhances lung fibrosis in mice. Nat Commun. 2018;9(1):5363.

40. Furusawa H, Cardwell JH, Okamoto T, Walts AD, Konigsberg IR, Kurche JS, et al. Chronic Hypersensitivity Pneumonitis, an Interstitial Lung Disease with Distinct Molecular Signatures. Am J Respir Crit Care Med. 2020;202(10):1430-44.

41. Dubois A, Ginet C, Furstoss N, Belaid A, Hamouda MA, El Manaa W, et al. Differentiation inducing factor 3 mediates its anti-leukemic effect through ROS-dependent DRP1-mediated mitochondrial fission and induction of caspase-independent cell death. Oncotarget. 2016;7(18):26120-36.

42. Ikeda Y, Shirakabe A, Maejima Y, Zhai P, Sciarretta S, Toli J, et al. Endogenous Drp1 mediates mitochondrial autophagy and protects the heart against energy stress. Circ Res. 2015;116(2):26478.

43. Karbowski M, Lee YJ, Gaume B, Jeong SY, Frank S, Nechushtan A, et al. Spatial and temporal association of Bax with mitochondrial fission sites, Drp1, and Mfn2 during apoptosis. J Cell Biol. 
2002;159(6):931-8.

44. Wagers S, Lundblad LK, Ekman M, Irvin CG, Bates JH. The allergic mouse model of asthma: normal smooth muscle in an abnormal lung? J Appl Physiol (1985). 2004;96(6):2019-27.

45. Chan YL, Wang B, Chen H, Ho KF, Cao J, Hai G, et al. Pulmonary inflammation induced by low-dose particulate matter exposure in mice. Am J Physiol Lung Cell Mol Physiol. 2019;317(3):L424-I30.

46. Zhang L, Ma C, Wang X, He S, Li Q, Zhou Y, et al. Lipopolysaccharide-induced proliferation and glycolysis in airway smooth muscle cells via activation of Drp1. J Cell Physiol. 2019;234(6):925563.

47. Delmotte P, Marin Mathieu N, Sieck GC. TNFa induces mitochondrial fragmentation and biogenesis in human airway smooth muscle. Am J Physiol Lung Cell Mol Physiol. 2021;320(1):L137-I51.

48. Zhao CC, Xu J, Xie QM, Fan XY, Fei GH, Wu HM. Apolipoprotein E negatively regulates murine allergic airway inflammation via suppressing the activation of NLRP3 inflammasome and oxidative stress. Int Immunopharmacol. 2020;81:106301.

49. Aravamudan B, Kiel A, Freeman M, Delmotte P, Thompson M, Vassallo R, et al. Cigarette smokeinduced mitochondrial fragmentation and dysfunction in human airway smooth muscle. Am J Physiol Lung Cell Mol Physiol. 2014;306(9):L840-54.

50. Huang Q, Zhan L, Cao H, Li J, Lyu Y, Guo X, et al. Increased mitochondrial fission promotes autophagy and hepatocellular carcinoma cell survival through the ROS-modulated coordinated regulation of the NFKB and TP53 pathways. Autophagy. 2016;12(6):999-1014.

51. Guido C, Whitaker-Menezes D, Lin Z, Pestell RG, Howell A, Zimmers TA, et al. Mitochondrial fission induces glycolytic reprogramming in cancer-associated myofibroblasts, driving stromal lactate production, and early tumor growth. Oncotarget. 2012;3(8):798-810.

52. Battaglia CR, Cursano S, Calzia E, Catanese A, Boeckers TM. Corticotropin-releasing hormone (CRH) alters mitochondrial morphology and function by activating the NF-kB-DRP1 axis in hippocampal neurons. Cell Death Dis. 2020;11(11):1004.

53. Gao F, Reynolds MB, Passalacqua KD, Sexton JZ, Abuaita BH, O'Riordan MXD. The Mitochondrial Fission Regulator DRP1 Controls Post-Transcriptional Regulation of TNF-a. Frontiers in Cellular and Infection Microbiology. 2021;10(843).

54. Liu R, Xu F, Bi S, Zhao X, Jia B, Cen Y. Mitochondrial DNA-Induced Inflammatory Responses and Lung Injury in Thermal Injury Murine Model: Protective Effect of Cyclosporine-A. Journal of Burn Care Research. 2019;40(3):355-60.

55. Zhang Q, Raoof M, Chen Y, Sumi Y, Sursal T, Junger W, et al. Circulating Mitochondrial DAMPs Cause Inflammatory Responses to Injury. Nature. 2010;464(7285):104-7.

56. Hepokoski M, Wang J, Li K, Li Y, Gupta P, Mai T, et al. Altered lung metabolism and mitochondrial DAMPs in lung injury due to acute kidney injury. American Journal of Physiology-Lung Cellular and Molecular Physiology.0(0):null.

57. Ma J, Rubin BK, Voynow JA. Mucins, Mucus, and Goblet Cells. Chest. 2018;154(1):169-76. 
58. Zuo WL, Shenoy SA, Li S, O'Beirne SL, Strulovici-Barel Y, Leopold PL, et al. Ontogeny and Biology of Human Small Airway Epithelial Club Cells. Am J Respir Crit Care Med. 2018;198(11):1375-88.

59. Lampert MA, Orogo AM, Najor RH, Hammerling BC, Leon LJ, Wang BJ, et al. BNIP3L/NIX and FUNDC1-mediated mitophagy is required for mitochondrial network remodeling during cardiac progenitor cell differentiation. Autophagy. 2019;15(7):1182-98.

60. Ludikhuize MC, Meerlo M, Gallego MP, Xanthakis D, Burgaya Julià M, Nguyen NTB, et al. Mitochondria Define Intestinal Stem Cell Differentiation Downstream of a FOXO/Notch Axis. Cell Metab. 2020;32(5):889-900.e7.

61. Sênos Demarco R, Jones DL. Mitochondrial fission regulates germ cell differentiation by suppressing ROS-mediated activation of Epidermal Growth Factor Signaling in the Drosophila larval testis. Sci Rep. 2019;9(1):19695.

62. Wasiak S, Zunino R, McBride HM. Bax/Bak promote sumoylation of DRP1 and its stable association with mitochondria during apoptotic cell death. J Cell Biol. 2007;177(3):439-50.

63. Frank S, Gaume B, Bergmann-Leitner ES, Leitner WW, Robert EG, Catez F, et al. The role of dynaminrelated protein 1, a mediator of mitochondrial fission, in apoptosis. Dev Cell. 2001;1(4):515-25.

64. Liu X, Zhao X, Li X, Lv S, Ma R, Qi Y, et al. PM(2.5) triggered apoptosis in lung epithelial cells through the mitochondrial apoptotic way mediated by a ROS-DRP1-mitochondrial fission axis. J Hazard Mater. 2020;397:122608.

65. Wang K, Zhan Y, Chen B, Lu Y, Yin T, Zhou S, et al. Tubeimoside l-induced lung cancer cell death and the underlying crosstalk between lysosomes and mitochondria. Cell Death Dis. 2020;11(8):708.

66. Kyung SY, Kim YJ, Son ES, Jeong SH, Park JW. The Phosphodiesterase 4 Inhibitor Roflumilast Protects against Cigarette Smoke Extract-Induced Mitophagy-Dependent Cell Death in Epithelial Cells. Tuberc Respir Dis (Seoul). 2018;81(2):138-47.

67. Cereghetti GM, Costa V, Scorrano L. Inhibition of Drp1-dependent mitochondrial fragmentation and apoptosis by a polypeptide antagonist of calcineurin. Cell Death Differ. 2010;17(11):1785-94.

68. Lee Y, Lee HY, Hanna RA, Gustafsson ÅB. Mitochondrial autophagy by Bnip3 involves Drp1-mediated mitochondrial fission and recruitment of Parkin in cardiac myocytes. Am J Physiol Heart Circ Physiol. 2011;301(5):H1924-31.

69. Mizumura K, Cloonan SM, Nakahira K, Bhashyam AR, Cervo M, Kitada T, et al. Mitophagy-dependent necroptosis contributes to the pathogenesis of COPD. J Clin Invest. 2014;124(9):3987-4003.

70. Zhang Y, Do DC, Hu X, Wang J, Zhao Y, Mishra S, et al. CaMKII oxidation regulates cockroach allergen-induced mitophagy in asthma. J Allergy Clin Immunol. 2021;147(4):1464-77.e11.

71. Zhang Y, Tang HM, Liu CF, Yuan XF, Wang XY, Ma N, et al. TGF- $\beta 3$ Induces Autophagic Activity by Increasing ROS Generation in a NOX4-Dependent Pathway. Mediators Inflamm. 2019;2019:3153240.

72. Wu G, Yang L, Xu Y, Jiang X, Jiang X, Huang L, et al. FABP4 induces asthmatic airway epithelial barrier dysfunction via ROS-activated FoxM1. Biochem Biophys Res Commun. 2018;495(1):1432-9. 
73. Lee HY, Lee GH, Kim HK, Chae HJ. Platycodi Radix and its active compounds ameliorate against house dust mite-induced allergic airway inflammation and ER stress and ROS by enhancing antioxidation. Food Chem Toxicol. 2019;123:412-23.

74. Lee AJ, Lim JW, Kim H. Ascorbic Acid Suppresses House Dust Mite-Induced Expression of Interleukin8 in Human Respiratory Epithelial Cells. J Cancer Prev. 2021;26(1):64-70.

\section{Figures}

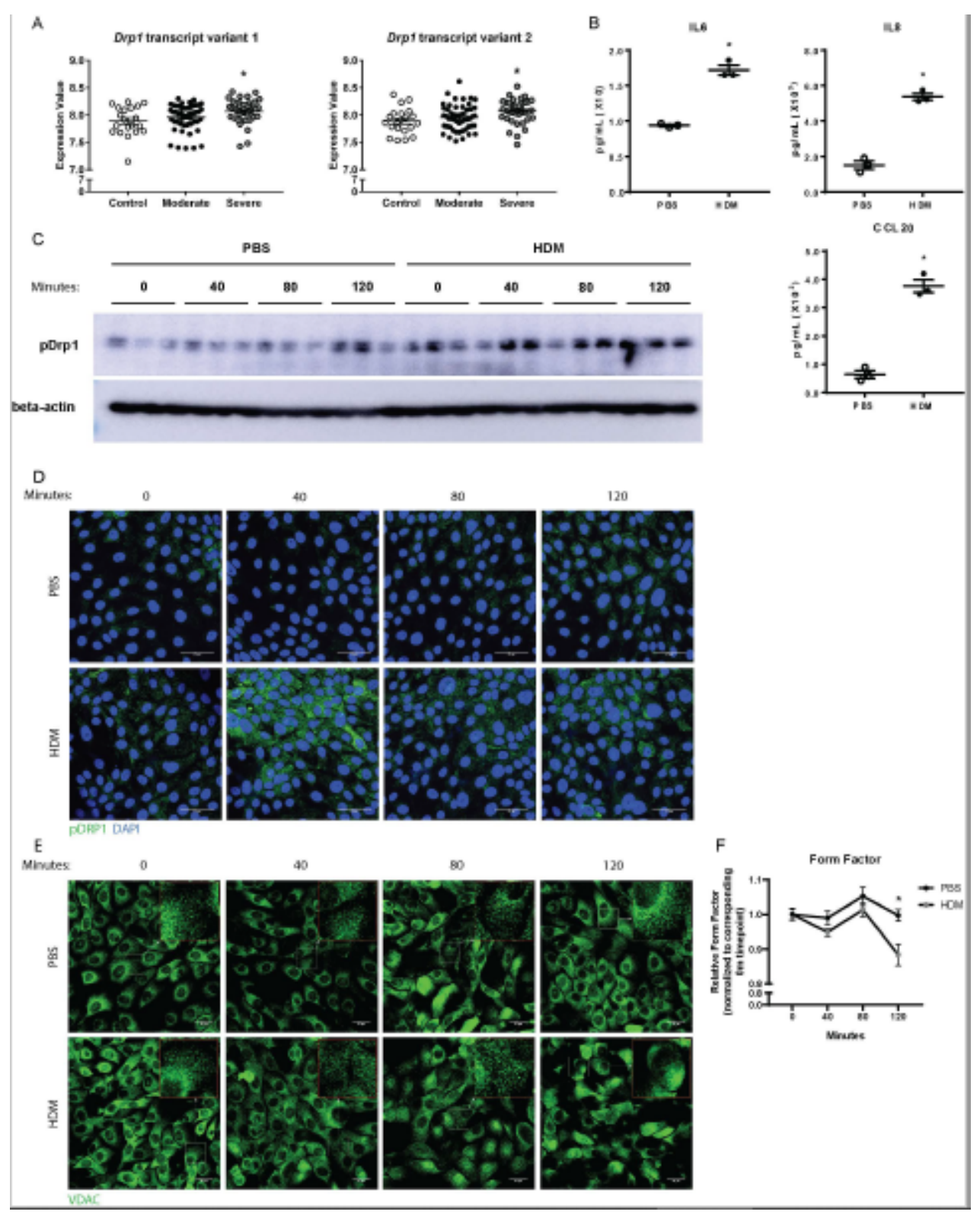

Figure 1

HDM induces mitochondrial fission in human bronchial epithelial cells. (A) human microarray data of Drp1 transcripts 1 and 2 from healthy controls, and patients with moderate and severe asthma. (B) ELISAs on supernatants from cultured HBECs, $n=3$ per group. (C) Western blot analysis of pDRP1 in whole cell lysates, $n=3$ per group. (D) Representative images of immunofluorescence of pDRP1 in fixed 
HBECs. (E) Representative images from immunofluorescence of VDAC in fixed HBECs. (F) Quantification of mitochondrial networking based on VDAC mitochondrial immunofluorescence, form factor calculation with $n=5$ images per group at $60 x$ magnification. $(B, C \& F) * P<0.05$ vs. PBS group. Error bars represent mean \pm SEM.

A

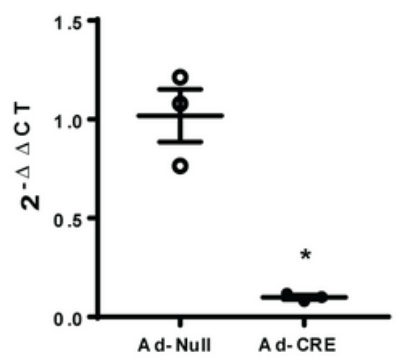

C Mitochondrial Networking

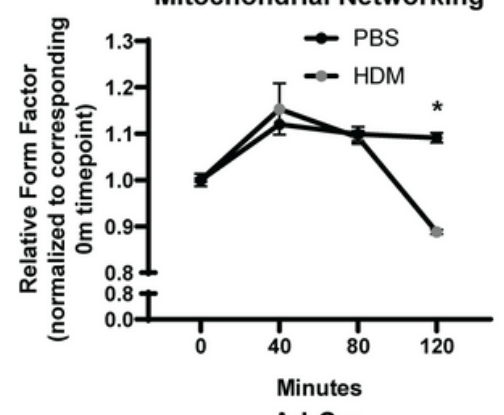
Ad-Cre Mitochondrial Networking

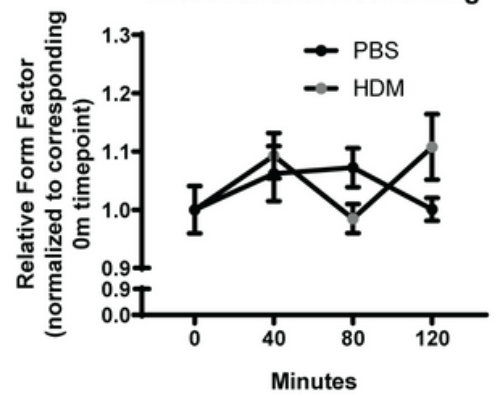

D

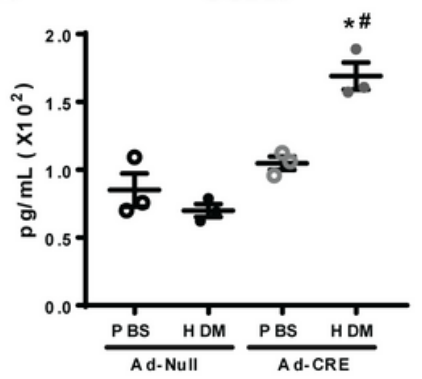

B Minutes:

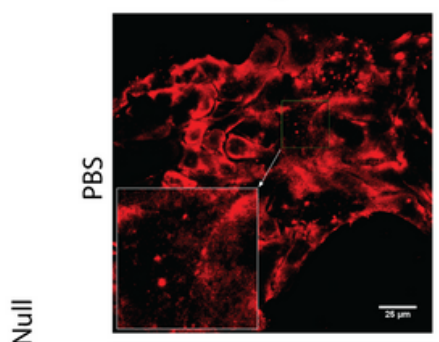

$\sum_{i}^{\frac{1}{2}}$

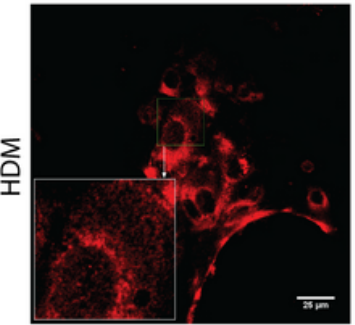

Minutes:

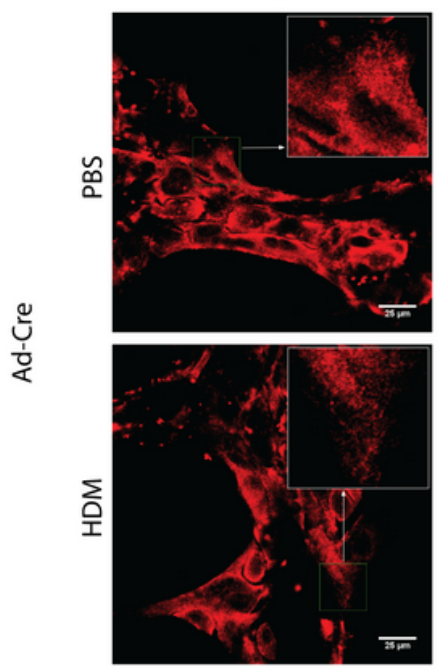

CXCL1

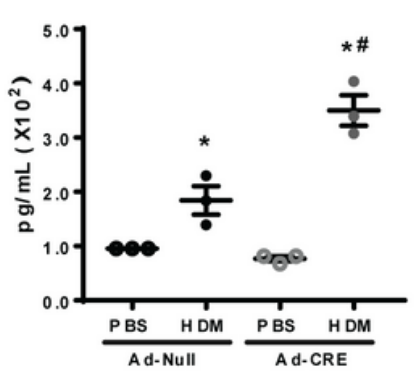

40
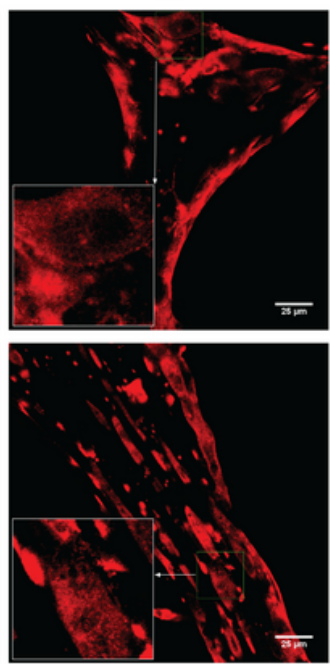

40
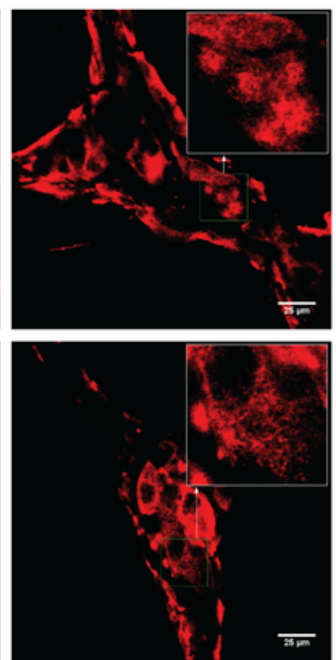

G CS F
80
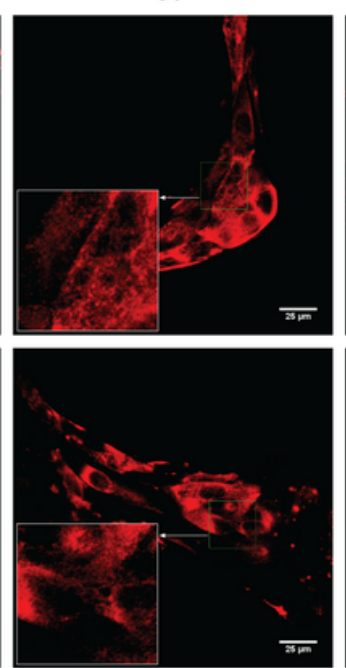

80
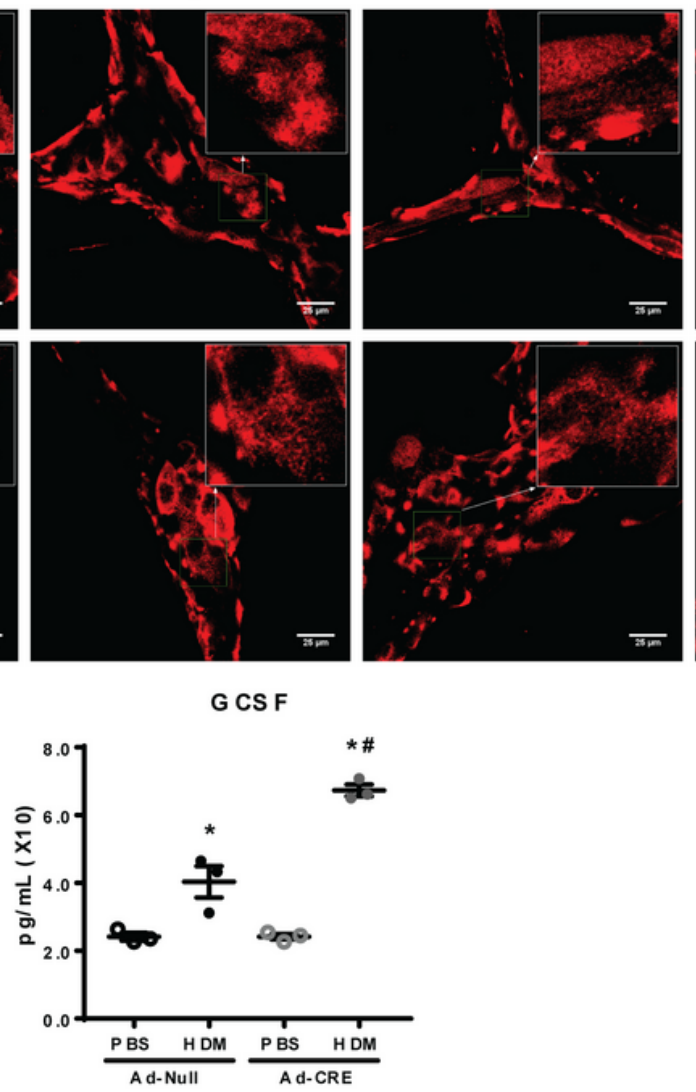

120
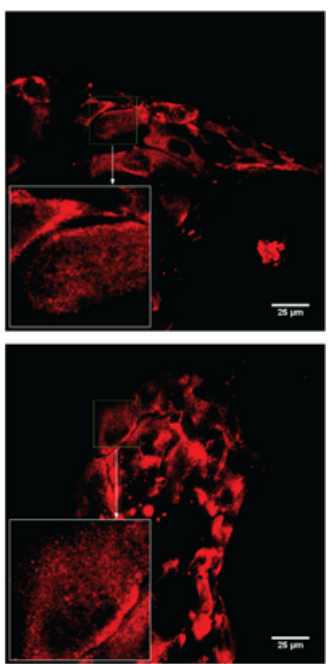

120
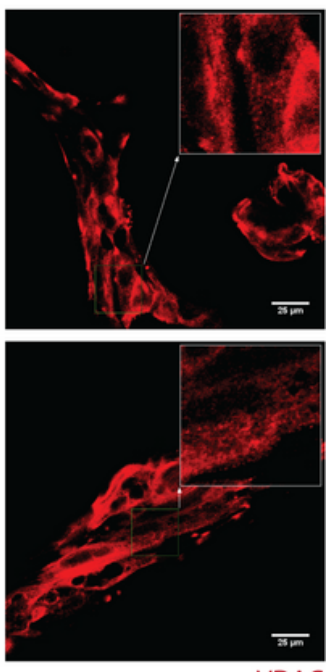

VDAC

Figure 2

Drp1 deletion impairs mitochondrial fission and enhances the mouse epithelial pro-inflammatory response to HDM. (A) RT-qPCR of Drp1 following Cre-induced deletion in cultured MTECs, $n=3$ per group. (B) Representative images of immunofluorescence of VDAC in fixed MTECs. (C) Quantification of 
mitochondrial networking based on VDAC immunofluorescence, form factor calculation with Null $n=3$ images per group, Cre $n=6-10$ images per group at $60 x$ magnification. (D) ELISAs on supernatants from MTECs, $n=3$ per group. $(A, C, \& D) * P<0.05$ vs. corresponding PBS group. (D) \#P<0.05 vs. Ad-null HDM group. Error bars represent mean \pm SEM.

A

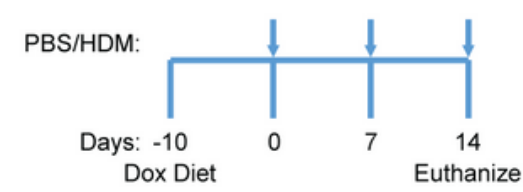

D

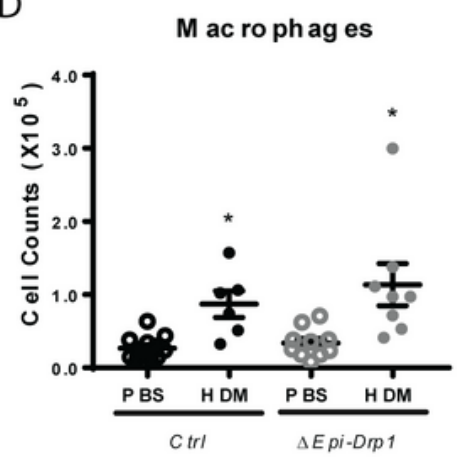

$E$

Pro-inflammatory

Chemokines

CXCL1

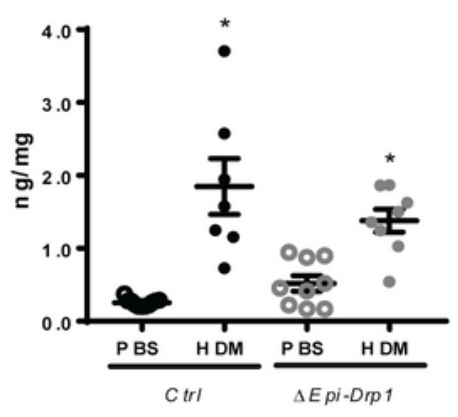

E ot ax in -1

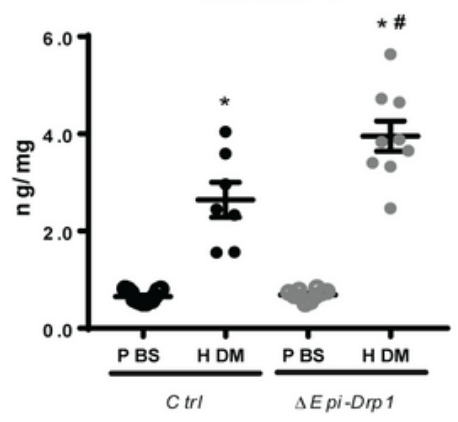

B

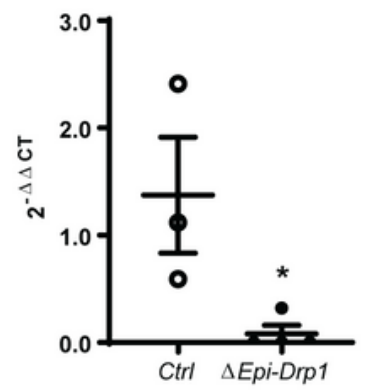

Epithelial Drp1 mRNA
$\mathrm{N}$ eu tr ophils

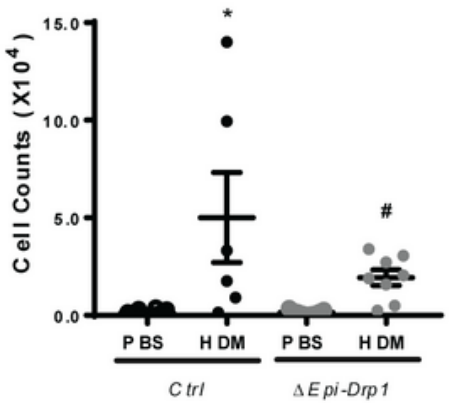

Pro-inflammatory Cytokines

IL- 6

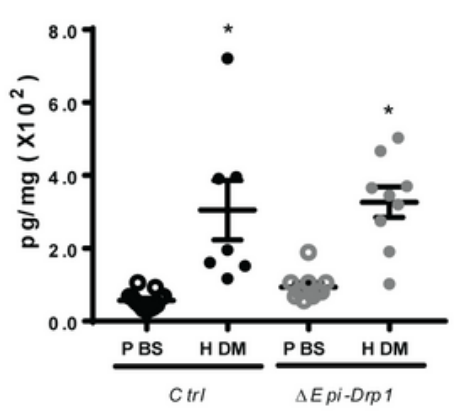

IL- 33

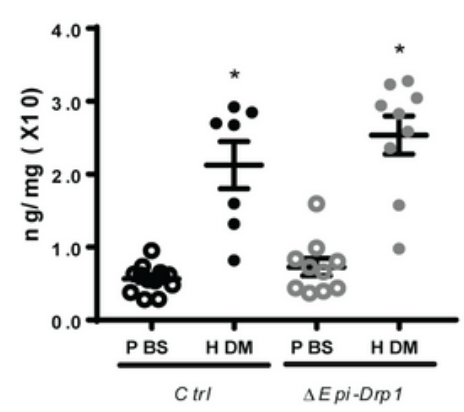

C Drp1 mRNA

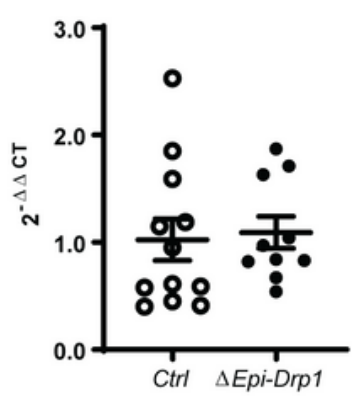

E os in ophi Is
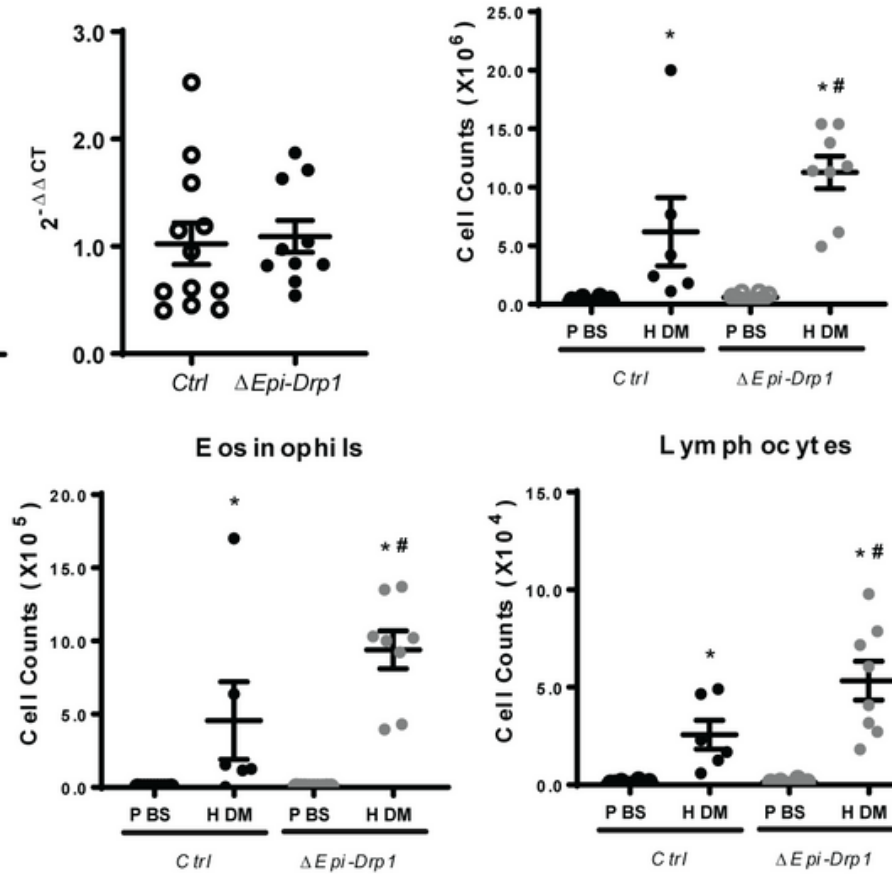

L ym ph oc yt es

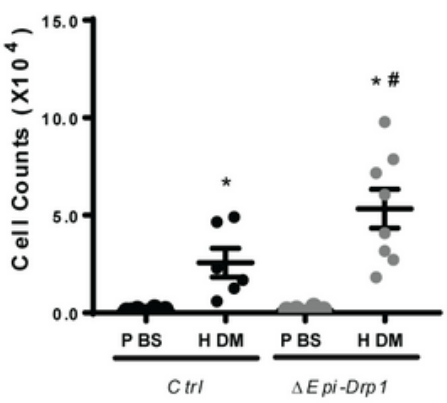

Th2 Adaptive

Cytokines

IL-4

IL- 13
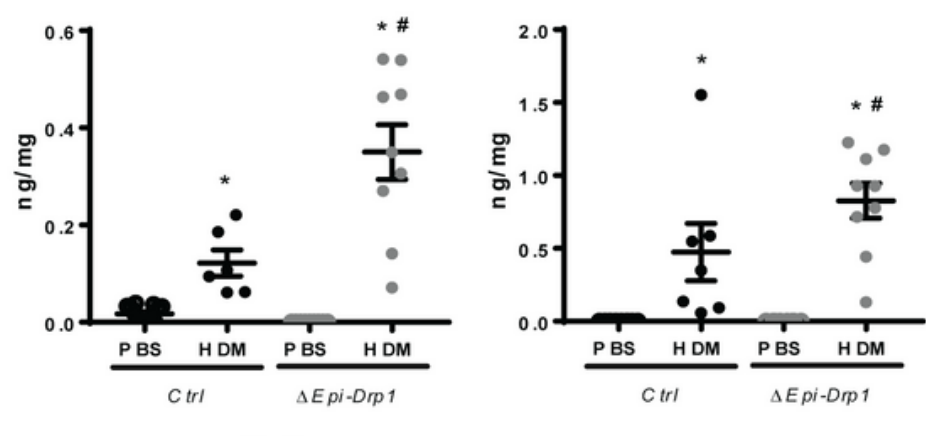

IL- 5

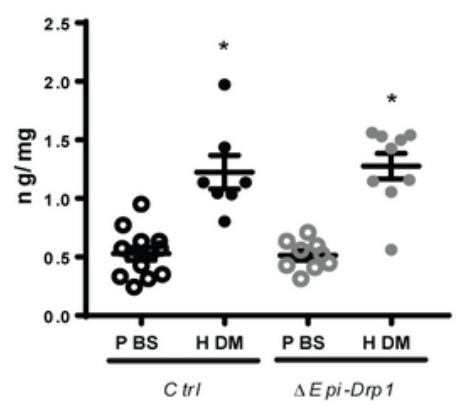

Figure 3 
Epithelial specific Drp1 deletion enhances inflammatory cell migration to mouse lungs in response to HDM. (A) Deletion and HDM exposure protocol. (B) RT-qPCR assessment of Drp1 in lung epithelial lysates, $n=3$ mice per group, and whole lung lysates, $n=10-12$ mice per group from 2 experiments. (C) Total inflammatory cells present in the BALF, $n=6-12$ mice per group from 2 experiments. (D) Inflammatory cell-specific totals in the BALF, $\mathrm{N}=6-12$ mice per group from 2 experiments. (E) ELISAs of inflammatory cytokines and chemokines in whole lung lysates, $n=6-13$ mice per group from 2 experiments. (B-E) * $P<0.05$ vs. corresponding PBS group, $\# P<0.05$ vs. Ctrl HDM group. Error bars represent mean \pm SEM. 
A

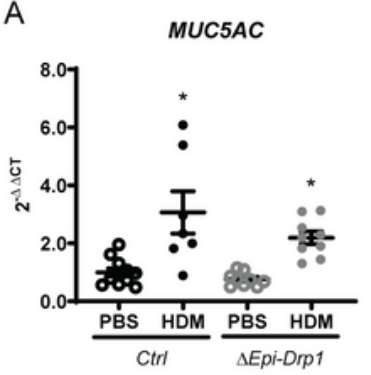

B
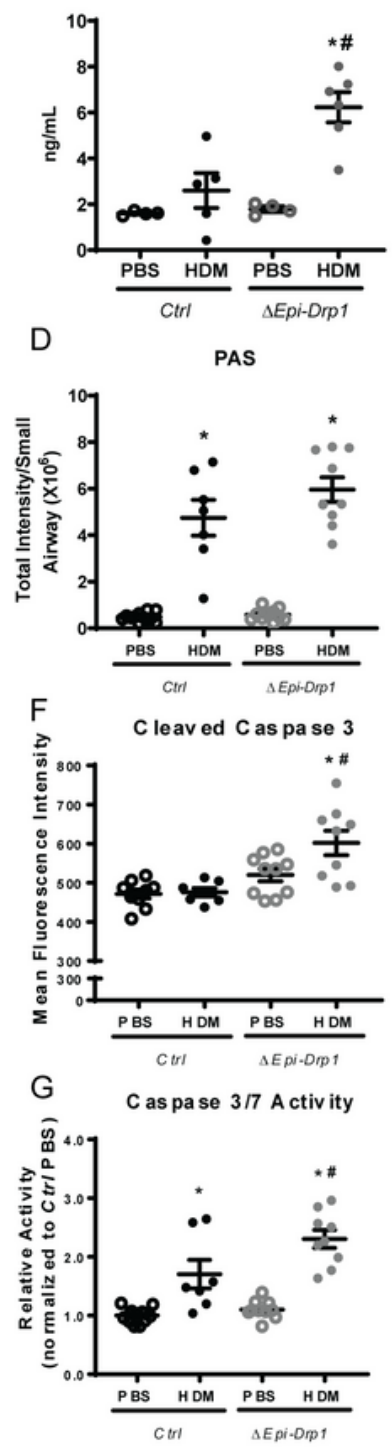

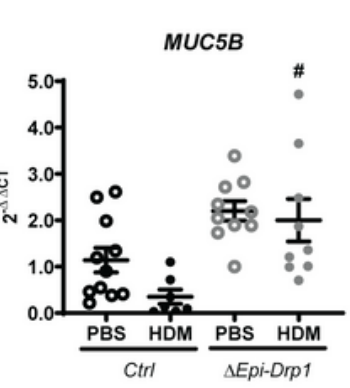

MUC5B

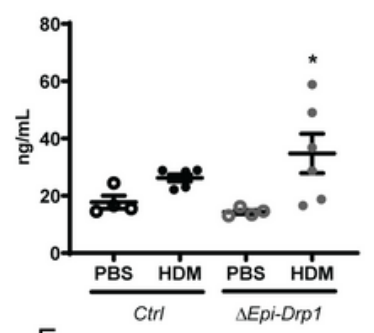

E
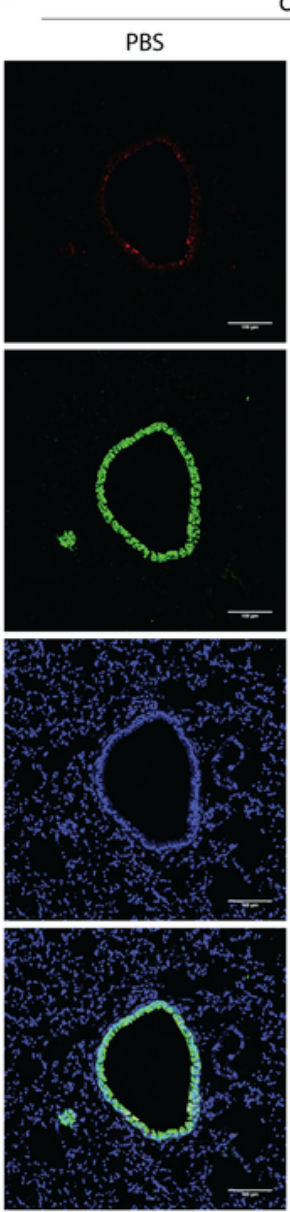

Cleaved Cas3 CC10 DAPI
C

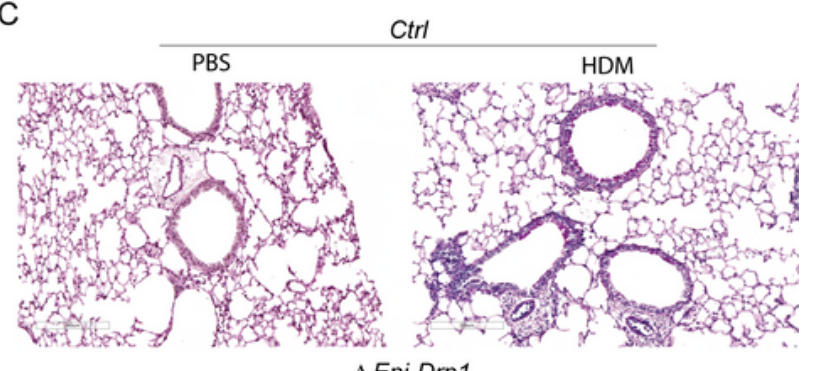

$\triangle$ Epi-Drp1
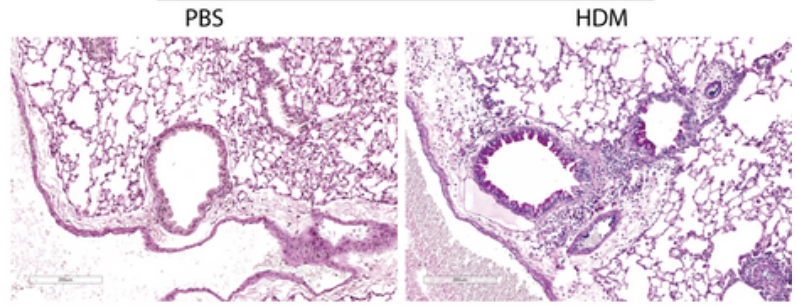

HDM
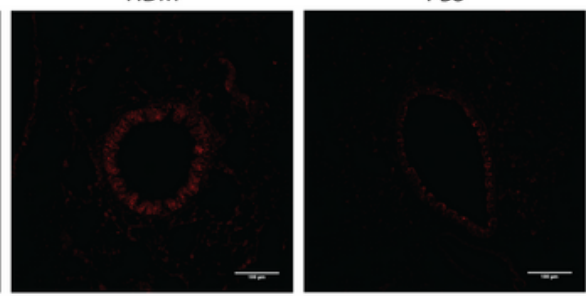

$\Delta$ Epi-Drp1
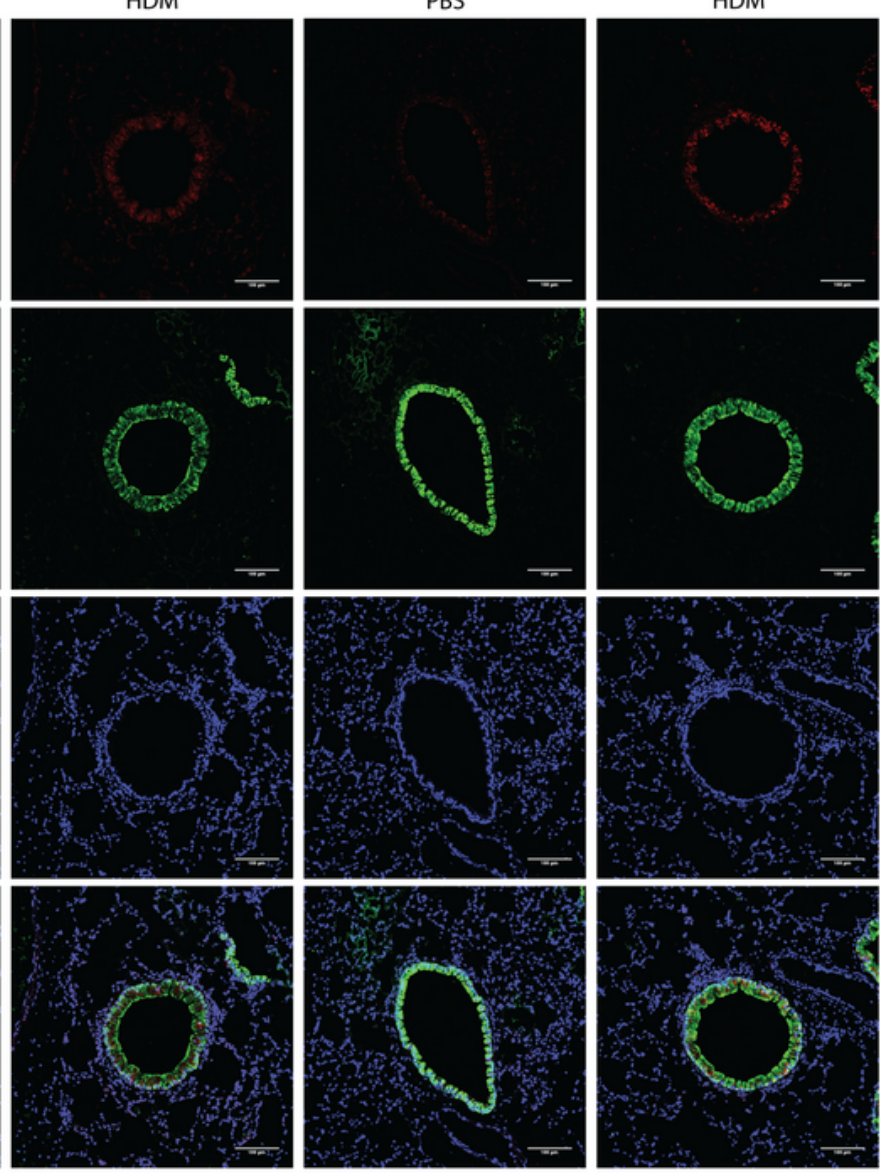

Figure 4

Epithelial specific Drp1 deletion enhances mucin metaplasia and epithelial cell death following HDM exposure. (A) RT-qPCR of mucins in whole lung lysates, $n=6-12$ mice per group from 2 experiments. (B) ELISAs of mucins in the BALF, $n=6-12$ mice per group from 2 experiments. (C) Representative images of PAS staining in lung tissue sections. (D) Quantification of PAS staining, $n=7-12$ mice per group from 2 experiments. (E) Representative immunofluorescence images of Cleaved Caspase 3 (Cleaved Cas3) and 
CC10. (F) Quantification of mean fluorescence intensity (MFI) of Cleaved Cas3 in immunofluorescence images, $n=7-10$ mice per group from 2 experiments. (G) Luminescence activity assay of Caspases 3 and 7 (Caspase Glo Assay) in whole lung lysates, $n=7-13$ mice per group from 2 experiments. (A, B, D, F \& G) ${ }^{*} \mathrm{P}<0.05$ vs. corresponding PBS group, $\# \mathrm{P}<0.05$ vs. Ctrl HDM group. Error bars represent mean $\pm \mathrm{SEM}$.

A

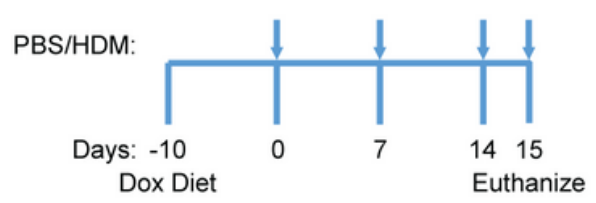

B
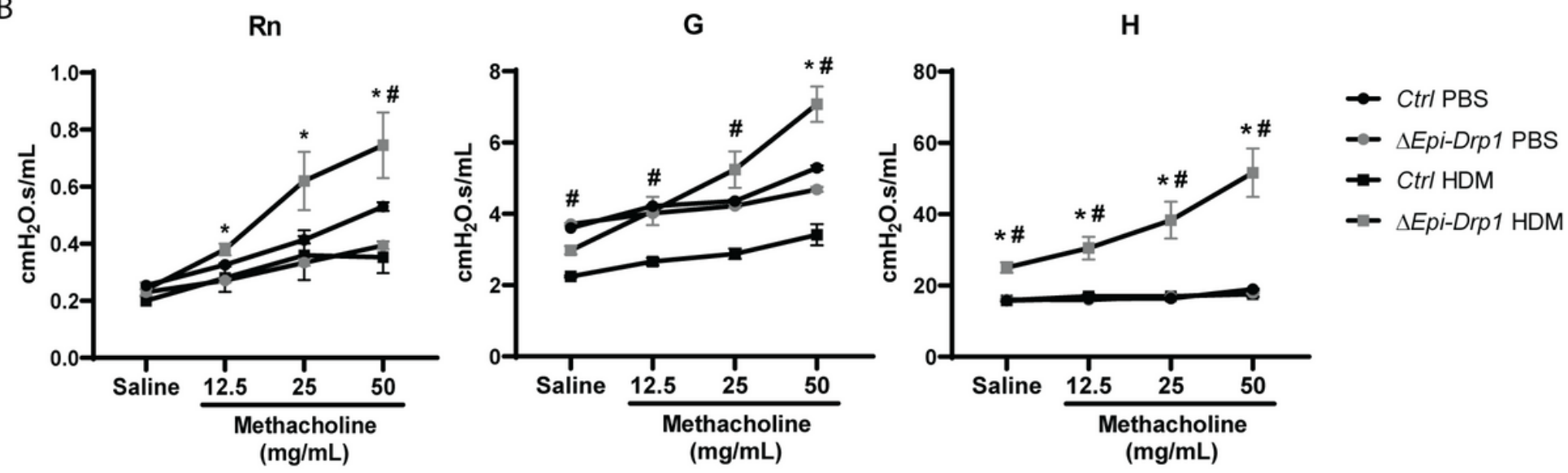

Figure 5

Epithelial specific Drp1 deletion enhances AHR in mouse lungs in response to HDM. (A) Deletion and HDM exposure protocol. (B) Results from AHR, $n=5$ mice per group from 2 experiments. (B) $* P<0.05$ vs. corresponding PBS group, \#P<0.05 vs. Ctrl HDM group. Error bars represent mean \pm SEM.

\section{Supplementary Files}

This is a list of supplementary files associated with this preprint. Click to download.

- FigureS1.tif 